\title{
Formação de estudantes da área da saúde em práticas integrativas e complementares
}

\section{Training health students in integrative and complementary practices}

\author{
Débora Regina Goecks ${ }^{1}$, Lisoni Muller Morsch ${ }^{1}$, Chana de Medeiros da Silva ${ }^{1}$ \\ 1 - Universidade de Santa Cruz do Sul - UNISC, Santa Cruz do Sul, RS, Brasil.
}

lmorsch@unisc.br

\section{RESUMO}

Objetivo: identificar qual o conhecimento, formação e interesse dos acadêmicos da área da saúde sobre Práticas Integrativas e Complementares (PIC). Método: a coleta ocorreu no período de setembro a outubro de 2018 por meio de um questionário estruturado online, enviado ao e-mail institucional da amostra. Participaram da pesquisa, 130 acadêmicos dos semestres iniciais $\left(1^{\circ}\right.$ ao $\left.3^{\circ}\right)$ e finais $\left(8^{\circ}\right.$ ao $10^{\circ}$ ). Resultados: houve participação de acadêmicos de nove cursos da área da saúde, 33 dos semestres iniciais e 97 dos semestres finais, predominando nessa população, o sexo feminino $(88,46 \%)$. Houve diferença significativa ( $<<0,05)$ entre semestres iniciais e finais quanto a "ouvir falar em PIC", $92,9 \%$ apontaram interesse no desenvolvimento destas práticas, porém não houve diferença significativa entre os semestres. As práticas apontadas como interesse em implementar e desenvolver estão o Reiki (44,9\%), Yoga (41,9\%), Meditação (42,4\%), Homeopatia (33,3\%) e Terapia de Florais (32,3\%). A participação em eventos/capacitações demonstrou diferença significativa $(p<0,05)$ entre semestres. Quanto a regulamentação das PIC, 54\% a desconhecem. Quanto ao exercício do profissional, se é exigida formação específica, 34,5\% declararam que sim. Da implementação das PIC no currículo, 87,9\% consideram importante para formação. Sobre a principal contribuição das PIC para o paciente, 97,5\%

Palavras-chave: apontaram como medida de promoção, prevenção e tratamento. Conclusão: estes resultados permitem

Terapias Complementares; concluir que os acadêmicos desejam que estas práticas sejam incluídas na sua formação, tornando-se Política de Saúde; Estudantes necessário o interesse e planejamento da universidade na inclusão destas no currículo dos acadêmicos de Ciências da Saúde. da área da saúde.

\begin{abstract}
Objective: identify the knowledge, education and interest of health academics about Integrative and Complementary Practices (ICP). Method: the collection took place from September to October 2018 and occurred through a structured online questionnaire, sent to the institutional e-mail of each sample. One hundred and thirty students from the initial (1st to 3rd) and final (8th to 10th) semesters participated in the research. Results: there was participation of academics from 9 courses in the health area, 33 from the initial semesters and 97 from the final semesters, predominantly female $(88.46 \%)$. There was a significant difference $(\mathrm{p}<0.05$ ) between initial and final semesters in terms of "hearing about ICP", $92.9 \%$ pointed out an interest in the development of these practices, but there was no significant difference between the semesters. Practices pointed out as interest in implementing and developing are Reiki (44.9\%), Yoga (41.9\%), Meditation (42.4\%), Homeopathy (33.3\%) and Flower Therapy (32.3 $\%)$. Participation in events / training showed significant difference $(\mathrm{p}<0.05)$ between semesters. As for the regulation of ICP, $54 \%$ are unaware of it. As for the professional practice, if specific training is required, $34.5 \%$ said yes. Of the implementation of ICP in the curriculum, $87.9 \%$ consider it important

Keywords: for training. About the main contribution of ICP to the patient, $97.5 \%$ pointed as a measure of promo-
\end{abstract} Complementary Therapies; tion, prevention and treatment. Conclusion: these results allow us to conclude that academics want Health Policy; Students; these practices to be included in their education, and the interest and planning of the university in their Health Occupations. inclusion in the curriculum of health scholars is necessary. 


\section{INTRODUÇÃO}

As Práticas Integrativas e Complementares (PIC) são técnicas milenares, utilizadas como recurso terapêutico ao longo de muitos anos, para fins de cuidados com a saúde, atuando de forma complementar, não apenas para determinada patologia, mas para proporcionar qualidade de vida à população. São denominadas pela Organização Mundial da Saúde (OMS) e Organização Pan-americana de Saúde (OPAS) como Medicina Tradicional (MT) e Medicina Complementar Alternativa (MCA), as quais envolvem abordagens de estímulo aos mecanismos naturais de prevenção de agravos e recuperação da saúde. ${ }^{1}$

A implantação das PIC no Sistema Único de Saúde (SUS) ainda é considerada um grande desafio, exigindo profissionais sensibilizados com o cuidado ampliado e humanizado na orientação de pacientes e na tomada de decisões, capazes de integrar diferentes saberes e práticas em saúde interagindo e colaborando com colegas que efetivamente adotem estas práticas como cuidados em saúde. ${ }^{2}$ Recentemente no $1^{\circ}$ Congresso Internacional de Práticas Integrativas e Saúde Pública - INTERCONGREPICS 2018 - foram oficialmente anunciadas à inclusão de dez novas PIC no SUS, fazendo do Brasil o país líder em oferecer estas modalidades para a atenção básica de saúde, voltada para prevenção de doenças como, por exemplo, hipertensão e depressão, totalizando vinte e nove práticas reconhecidas pelo SUS. ${ }^{3}$

Em alguns países, os programas de formação profissional em MCA no nível universitário estão inseridos no ensino profissional em saúde, principalmente em cursos de Medicina e Farmácia². No Brasil predominam os programas oferecidos na maioria das vezes em instituições privadas por meio de cursos de especialização, porém observa-se também uma tendência e crescimento da oferta de disciplinas em PIC nas diferentes graduações da área da saúde. ${ }^{4}$

É possível observar que estas práticas estão aos poucos sendo discutidas e estudadas na formação superior da área da saúde 5 . Percebe-se, portanto, a necessidade de verificar, junto aos acadêmicos da área da saúde qual o conhecimento, aceitação e interesse que apresentam pelas PIC, a fim de sensibilizá-los e ao mesmo tempo propor aos coordenadores de cursos da área da saúde a implementação e oferta de disciplinas curriculares ou cursos de extensão sobre a temática nas universidades. Frente ao exposto, este trabalho tem como objetivo identificar qual o conhecimento, aceitação e interesse dos acadêmicos da área da saúde sobre as PIC na Universidade de Santa Cruz do Sul (UNISC).

\section{MÉTODO}

Trata-se de um estudo com delineamento transversal, observacional e descritivo realizado com estudantes de nove cursos da área da saúde da UNISC (Biomedicina, Enfermagem, Estética e Cosmética, Farmácia, Fisioterapia, Medicina Veterinária, Nutrição, Psicologia e Serviço Social), no município de Santa Cruz do Sul, RS, durante o período de setembro a outubro de 2018. Os acadêmicos foram convidados a participar através do preenchimento de um questionário online do "Google Formulários", que lhes foi enviado pelo e-mail institucional e aos que aceitaram o Termo de Consentimento Livre e Esclarecido (TCLE), participaram da pesquisa. Como critérios de inclusão, foram considerados sujeitos da pesquisa, os acadêmicos com idade superior a 18 anos, de ambos os sexos e que estivessem cursando semestres iniciais $\left(1^{\circ}\right.$ ao $3^{\circ}$ semestre $)$ e finais $\left(8^{\circ}\right.$ ao 10) dos cursos da área da saúde.

O questionário estruturado foi composto por 10 perguntas objetivas, relacionadas ao conhecimento, formação e interesse das PIC. A análise descritiva dos dados e a associação entre as variáveis pelo teste qui-quadrado $(p<0,05)$ foram realizadas através do software SPSS, versão 20.0. O estudo foi submetido e aprovado pelo Comitê de Ética em Pesquisa da Universidade de Santa Cruz do Sul, sob parecer $n^{\circ}$ 2.837.222.

\section{RESULTADOS}

A amostra foi composta por 130 estudantes dos cursos da área da saúde ( $\mathrm{n}=9), 88,46 \%(\mathrm{n}=115)$ eram do sexo feminino e $11,54 \%(n=15)$, do sexo masculino. A distribuição dos estudantes por curso pode ser visualizada na Figura 1. Os três cursos com maior número de estudantes participantes foram os da Farmácia $(n=47)$, Estética e Cosmética $(n=24)$, Nutrição $(n=19)$ e Psicologia $(n=19)$.

Embora muitos estudantes tenham relatado o conhecimento de alguma prática integrativa e complementar, houve uma diferença significativa $(p<0,05)$ entre semestres iniciais e finais quanto a "ouvir falar em PIC", destacando-se para o Reiki (82,3\%), Meditação (81,8\%), Yoga $(77,8 \%)$, 


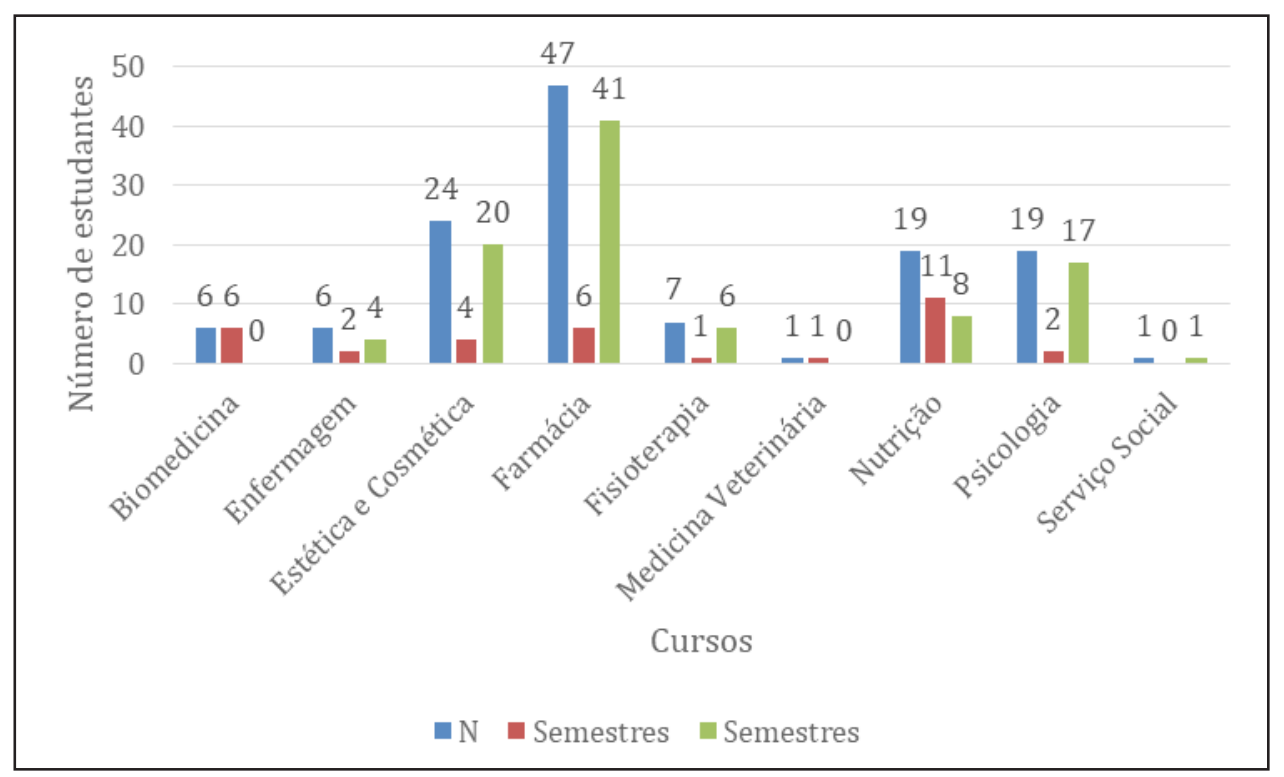

Figura 1 - Representação gráfica da distribuição dos estudantes conforme o curso e semestre.

Homeopatia (71,2\%) e Aromaterapia (68,2\%).

Sobre a utilização das PIC, não houve diferença significativa entre os semestres, em que $65,7 \%$ dos estudantes declararam já utilizar alguma PIC. Dentre os profissionais citados pelos estudantes que trabalham com as PIC foram apontados os da Fisioterapia $(31,3 \%)$, Enfermagem $(28,8 \%)$, Farmácia $(22,7 \%)$, Psicologia $(18,7 \%)$ e Esteticista (14,1\%). Sobre a importância da implantação destas práticas no SUS não houve diferença significativa entre os semestres e, todos (100\%) estudantes indicaram ser importante. Quando questionados se durante a graduação tiveram conhecimento ou acesso a alguma PIC, houve uma diferença significativa $(p<0,05)$ entre os semestres, aqueles dos semestres finais tiveram mais acesso que os inicias, em que se destacam a Homeopatia (40,9\%), Fitoterapia (38,9\%), Reiki (34,8\%), Aromaterapia $(25,8 \%)$ e Meditação (25,3\%).

Sobre o interesse na oferta de disciplinas, cursos e palestras sobre as PIC durante a graduação não houve diferença significativa entre os semestres, porém $66,7 \%$ do total de estudantes apontaram interesse especialmente para o Reiki $(44,9 \%)$, Yoga (41,9\%), Meditação (42,4\%), Homeopatia (33,3\%) e Terapia de Florais (32,3\%). Sobre a implementação das PIC no currículo do seu curso, 87,9\% acham que estas são de suma importância para formação acadêmica e para vida profissional, $7,1 \%$ não acham necessária a implementação das PIC no currículo e $5,1 \%$ acham que são importantes na formação, porém destacaram que não agregariam conhecimento a vida profissional.

Questionados quanto a participação em eventos/capacitações sobre PIC, houve diferença significativa $(p<0,05)$ entre os semestres, sendo que seminários ou palestras teve maior destaque, conforme demonstrado na Tabela 1.

Sobre a regulamentação das PIC, 39,4\% declararam que são regulamentadas, $6,6 \%$ não e $54 \%$ não sabem se elas são. Quanto ao exercício do profissional da saúde nas PIC, se é exigida formação específica, $34,5 \%$ declaram que sim, $5,1 \%$ não, $33,5 \%$ em algumas áreas e 34,5\% não sabem. Sobre

Tabela 1 -Distribuição das frequências absolutas sobre o conhecimento e aceitação das Práticas Integrativas durante a graduação. Brasil - 2018.

\begin{tabular}{|c|c|c|c|c|c|c|}
\hline \multirow{2}{*}{ Variável } & \multicolumn{2}{|c|}{ Sim } & \multicolumn{2}{|c|}{ Não } & \multicolumn{2}{|c|}{ Nenhuma } \\
\hline & Inicial & Final & Inicial & Final & Inicial & Final \\
\hline Você já ouviu falar nas Práticas Integrativas e Complementares (PICs)? & 32 & 91 & 5 & 2 & 0 & 0 \\
\hline Já utilizou alguma terapia alternativa? & 23 & 66 & 14 & 27 & 0 & 0 \\
\hline Conhece alguém que trabalhe com alguma destas práticas? & 29 & 81 & 8 & 12 & 0 & 0 \\
\hline Acha importante a implantação destas práticas no SUS? & 37 & 89 & 0 & 4 & 0 & 0 \\
\hline $\begin{array}{l}\text { Durante a graduação, teve conhecimento ou acesso a alguma das Práticas } \\
\text { Integrativas e Complementares (PICs)? }\end{array}$ & 23 & 81 & 14 & 12 & 0 & 0 \\
\hline Tem interesse que estas práticas sejam desenvolvidas durante a graduação? & 36 & 87 & 1 & 4 & 0 & 2 \\
\hline Já participou de evento/capacitação sobre PICs? & 12 & 54 & 25 & 39 & 0 & 0 \\
\hline
\end{tabular}


a utilização das PIC, 97,5\% apontaram como medida de promoção, prevenção e tratamento de doenças, melhorando a qualidade de vida do paciente, $2 \%$ como medida de tratamento e prevenção quando pacientes não tem acesso ao tratamento farmacológico convencional e $0,5 \%$ como medida paliativa, não trazendo nenhum benefício direto à saúde.

\section{DISCUSSÃO}

Na década de 70, os fundamentos, conceitos e práticas da Medicina Tradicional Chinesa e Indiana foram à base para a consolidação mundial das PIC, trazendo práticas milenares como a acupuntura, fitoterapia, termalismo, Shiatsu e massoterapias como medidas de tratamento e prevenção da saúde na atenção básica.

O uso das PIC no Brasil é estabelecido pela PNPIC - Política Nacional de Práticas Integrativas e Complementares, a portaria $\mathrm{n}^{\circ} 971$, publicada em 2006, disponibilizou cinco práticas ao Sistema Único de Saúde (SUS) em todo o país, em março de 2017 a portaria $\mathrm{n}^{\circ} 849$, inclui mais 14 práticas e em março de 2018 pela portaria $n^{\circ} 702$, foram introduzidas mais dez práticas, totalizando vinte e nove práticas disponíveis pelo SUS, fazendo com que o Brasil seja líder em oferecer estas modalidades para a atenção básica de saúde. ${ }^{3,6,7}$

Um novo modelo conceitual de comprometimento com o uso da MCA está sendo elaborado e estudado, baseado nos princípios da psicologia do paciente, a partir das necessidades, motivação e confiabilidade em valores de cuidado da saúde, revelando a importância do compromisso do paciente com a prática que lhe está sendo aplicada, melhorando seu potencial de aplicação, trazendo maiores benefícios, resultados positivos, tudo isso para melhorar o seu tratamento e sua qualidade de vida. ${ }^{8}$

O uso das PIC tem representado um grande benefício aos pacientes, principalmente por promoverem o alívio dos sintomas emocionais e psicológicos, especialmente daqueles pacientes que estão enfrentando alguma doença crônica, auxiliando-os no seu bem-estar e no processo de cura. $\mathrm{O}$ interesse e o reconhecimento médico por estas práticas já é notável, principalmente devido aos resultados positivos apresentados até o momento na saúde de muitos pacientes, promovendo cuidados de saúde física e mental, diminuindo inclusive os efeitos adversos de medicamentos. ${ }^{9}$

Muitas intervenções com a MCA, quiropraxia e terapias de massagem, trazem como evidência a capacidade individual de cada paciente em alcançar seu próprio equilíbrio físico restaurando sua própria saúde. Isto é possível pelo contexto deste tipo de intervenção, principalmente no que se refere à relação entre paciente e profissional, promovendo tratamentos individualizados, considerando um sistema completo de cuidado de saúde. ${ }^{10}$

Usuários de PIC relataram utilizar estas práticas não somente como medida terapêutica associada ao tratamento farmacológico, mas também para manter o seu bem-estar físico e mental, adquirir hábitos de vida saudáveis, melhorar a qualidade de vida, incorporando a MCA como um estilo de vida saudável, trazendo resultados positivos para a sua saúde. ${ }^{10}$

A procura por uma prática alternativa também se deve em muitos casos pelo alto custo da assistência privada associado com a precariedade de assistência prestada pelos serviços de saúde pública. ${ }^{11}$ Segundo a OMS em países mais desenvolvidos a procura por alternativas naturais para cuidados primários de saúde é muito maior devido à tradição cultural e pela dificuldade de acesso da população a tratamentos de alto custo. ${ }^{12}$ Vários municípios brasileiros já inseriram as PIC na terapêutica de pacientes que apresentam problemas de saúde como asma, rinite, dores musculares, insônia e depressão adotando práticas como acupuntura, bioenergética, homeopatia, meditação, yoga e massoterapia. ${ }^{5,13}$

No presente estudo é notável que a maior participação foi a de mulheres com $88,45 \%$ dos estudantes. Em outro estudo ${ }^{13}$ realizado com acadêmicos da área da saúde mostra também a maior prevalência para o sexo feminino $(66,7 \%)$, isso se deve ao fato das mulheres apresentarem maior interesse por estas práticas e por se preocuparem mais com as questões relacionados à saúde. Foi encontrada uma diferença significativa entre semestres iniciais e finais quanto a "ouvir falar em PIC" e, 94,9\% dos estudantes apontaram ter interesse no desenvolvimento das práticas durante a sua graduação. Assuntos sobre as PIC ainda são pouco desenvolvidos pelas universidades e destacam a necessidade de ampliar o acesso dos universitários às práticas que correspondam à sua formação tornando imprescindível o conhecimento das demandas da sociedade e do governo. Dentre as 
estratégias sugeridas estão as saídas a campo onde os estudantes realizam pesquisas sobre os assuntos e trazem a vivência do momento para ser discutida em sala de aula, ou a realização de palestras, oficinas, bem como, a oferta de disciplinas optativas e obrigatórias ao currículo. ${ }^{14}$ Tudo isto despertaria um maior interesse dos estudantes universitários em conhecer melhor as PIC e assim aplicá-las futuramente em sua vida profissional.

Um ponto que corrobora para o desenvolvimento das PICS na graduação dos estudantes da área da saúde é pelo fato da Lei de Diretrizes e Bases da Educação (LDB), permitir a flexibilidade dos currículos, onde está previsto que o atual profissional da saúde deve estar preparado para o trabalho coletivo num processo de cuidado da saúde, atendendo as prioridades de saúde da população, resolvendo o problema de saúde a nível individual como também coletivo. ${ }^{15}$

As diretrizes curriculares nacionais dos cursos da saúde descrevem eixos obrigatórios para a formação e perfil do egresso e dentre estes eixos encontra-se o do "cuidado em saúde" que visa reforçar conhecimentos, competências e habilidades de cuidado em saúde, PIC considerando a autonomia do ser humano, procurando terapias farmacológicas e não farmacológicas comprovadas cientificamente. Além disso, reforça que a atenção à saúde deve ser de acesso universal de forma a atender a todos e suas principais necessidades de saúde, como uma estratégia de promoção e proteção à saúde. ${ }^{16}$

\section{Algumas Práticas Complementares} (acupuntura, Reiki e massoterapia) incluídas em alguns currículos dos cursos de Enfermagem têm como propósito humanizar o auto-cuidado com o paciente..$^{5}$ Entretanto, a educação de nível superior brasileira ainda é carente destas práticas, é preciso que as universidades incluam no currículo dos cursos da área da saúde a abordagem sobre estas terapias, trazendo possibilidades de escolhas aos pacientes que precisam da assistência à saúde, podendo optar por estas práticas quando estiverem disponíveis. ${ }^{17}$

Os resultados demostram que $34,5 \%$ dos acadêmicos entrevistados não sabem da exigência do profissional da saúde ter uma formação específica para aplicabilidade das PIC. No Brasil, em meados de 1995, o Conselho Federal de Enfermagem emitiu o Parecer Normativo 004/95 aprovado na $239^{\text {a }}$ Reunião Ordinária realizada em 18 de julho de 1995, dispondo das terapias alternativas como Acupuntura, Iridologia,
Fitoterapia, Reflexologia, Quiropraxia e Massoterapia como práticas alternativas a serem realizadas pelo profissional da Enfermagem, mesmo assim, ainda são poucas instituições de ensino que oferecem disciplinas relacionadas as PIC para a formação do Enfermeiro. É importante salientar que o profissional da Enfermagem é o que passa a maior parte do tempo com o paciente, o que possibilita acompanhar de perto e avaliar os efeitos físicos e emocionais dessas práticas. $^{18}$

O Hidrotermalismo é uma prática muito estudada nos cursos de Fisioterapia, viabilizando o desenvolvimento da profissão através de um trabalho interdisciplinar criando estratégias em educação em saúde, promoção, prevenção e reabilitação beneficiando assim aquele paciente que procura esta prática como uma forma de recuperação em saúde. ${ }^{19} \mathrm{Em}$ 1980, o Conselho Federal de Medicina (CFM) reconheceu a Homeopatia como especialidade (Resolução CFM 1.000/80), na formação médica, em 1994 teve início a experiência do ensino da medicina homeopática na graduação médica da Universidade Federal Fluminense (UFF) Niterói (RJ). ${ }^{20}$ Desde então, multiplicaram-se os cursos de formação e especialização em Homeopatia, com o intuito de um olhar anatomoclínico para um olhar ampliado, possibilitando aos futuros profissionais a orientação de usuários dos serviços de saúde que desejam utilizar a Homeopatia de forma alternada, complementar ou integrada em seu tratamento. ${ }^{20} \mathrm{~A}$ inclusão da disciplina de Homeopatia no currículo da graduação dos cursos de Farmácia, por exemplo, tem como objetivo reformular a formação biomédica, trazendo um olhar mais ampliado para a integridade do cuidado da saúde, de forma a auxiliar no tratamento como também idealizar novas pesquisas, recriando um laboratórioescola de Homeopatia, comparando diferentes experiências, oferecendo fórum de discussões sobre o tema, criando um conjunto de modelos terapêuticos que permitem com que os estudantes criem uma maneira de fortalecer a integridade e o cuidado com o paciente. ${ }^{21}$

Quando questionados se durante a sua graduação tiveram conhecimento ou acesso a alguma das Práticas Integrativas e Complementares, foi encontrada diferença significativa $(p<0,05)$ entre semestres inicias e finais. Dentre as PIC mais abordadas na graduação destacam-se a homeopatia $40,9 \%$, fitoterapia $39,9 \%$ e reiki $34,8 \%$. Um estudo ${ }^{22}$ com acadêmicos do curso de Enfermagem de uma 
universidade privada demostrou que $59 \%$ dos alunos referiram nunca ter discutido sobre este assunto durante a formação acadêmica, e dos que tiveram, $71 \%$ respondeu que foi através da oferta de disciplinas como, por exemplo, Terapias Alternativas.

Embora muitos acadêmicos tenham demonstrado interesse em conhecer e estudar as PIC e incluí-las em seu currículo, outros citam a falta de evidências científicas. Um estudo ${ }^{23}$ realizado com universitários do curso de Farmácia da Universidade do Sudeste do Queensland na Austrália demonstrou que a maioria dos acadêmicos tem conteúdos sobre as MCA no seu currículo, porém a capacidade dos mesmos em aprender e aplicar estas práticas se deve ao seu interesse em aprofundar o conhecimento sobre as mesmas.

A expansão da MCA em todo mundo fez com que profissionais da área da saúde ampliassem seus conhecimentos sobre estas práticas para melhor aconselhar, orientar, enfrentar desafios, e melhorar a qualidade de vida de seus pacientes. ${ }^{24} \mathrm{Uma}$ forma de incorporar as PIC no currículo é oferecendo disciplinas optativas ou obrigatórias, trazendo para perto dos acadêmicos um conhecimento mais aprofundado para que no momento em que estiverem no mercado de trabalho possam aplicar e implementar estas práticas. ${ }^{2}$ Entretanto, palestras e cursos teóricos são formas mais rápidas e igualmente eficientes para despertar o interesse dos acadêmicos pelas PIC, mostrando seus benefícios ou malefícios, indicações ou contraindicações, desenvolvendo novas formas de tratamento e prevenção de doenças e outros agravos. ${ }^{13}$

A procura por uma metodologia de ensino ativa como saídas a campo, capacitações, desenvolvimento de pesquisas e posteriormente a apresentação dos resultados destas, promovem uma aprendizagem mais significativa e interessante para os acadêmicos, fazendo com que o mesmo vá à campo em busca de resultados e conhecimentos que enriqueçam o seu currículo e posteriormente possam aplicar as PIC conforme a necessidade e a disponibilidade que o serviço de saúde oferece. ${ }^{14}$ No ano de 2008 o Ministério da Saúde implantou o Centro de Especialidades de promoção da saúde incluindo as PIC para pacientes da Atenção Básica, desenvolvendo-as em grupo ou individualmente como formas de tratamento preventivo e curativo, oferecendo inicialmente práticas como, homeopatia, oficina de memória, acupuntura, dança e relaxamento. ${ }^{25}$ Quanto ao interesse destas práticas serem implantadas no SUS,
$96,92 \%$ dos estudantes considera importante, sendo 37 destes dos semestres inicias e 89 dos semestres finais.

A OMS incentiva a expansão das Medicinas Tradicional/Complementar e Alternativa nos países desenvolvidos, como é o caso do Brasil que se difere de outros países na oferta de PIC, pois predominam as práticas corporais, principalmente nos serviços públicos. Além disso, investimentos concentram-se nos níveis primários de atenção dando uma ênfase muito maior para as PIC, mostrando que existe uma grande diversidade nas atividades e de profissionais que realizam estas práticas. ${ }^{25}$

Dentre as PIC desenvolvidas e ofertadas pelo SUS, existem aquelas que são de natureza coletiva, que através de estratégias de intervenções em grupos visa criar uma rede de apoio a partir da troca de experiências de cada paciente, trazendo um sentimento de igualdade, contribuindo para fatores como redução do uso de medicações e surgimento de novas doenças, sendo a Estratégia de Saúde da Família (ESF) responsável por organizar uma equipe multiprofissional através do Núcleo de Apoio à Saúde da Família (NASF), o qual identifica as áreas estratégicas para as ações em PIC como, por exemplo, danças circulares, tenda do conto, arteterapia e grupos de conversa com mulheres. ${ }^{26}$ Considerando estes aspectos legais e de incentivo para oferta das PIC na Atenção Básica, torna-se imprescindível a discussão e reflexão das universidades no que diz respeito à formação dos estudantes da área da saúde nesta temática.

\section{CONCLUSÃO}

De acordo com os resultados obtidos é possível concluir que 94,9\% dos acadêmicos da área da saúde da Universidade de Santa Cruz do Sul do município de Santa Cruz do Sul, RS conhecem as PIC, assim como possuem interesse e aceitação em sua implementação nos cursos da área da saúde durante a graduação. Também foi verificado que, tanto os acadêmicos dos semestres inicias como finais consideram importante a implantação destas práticas no SUS.

Cerca de $66,2 \%$ dos acadêmicos que conhecem as PIC e já as utilizaram em algum momento, acreditam que a utilização destas práticas contribui com a promoção, prevenção e tratamento de doenças, melhorando a qualidade de vida do 
paciente, melhorando a qualidade de vida e a saúde do paciente. Portanto, conclui-se que os acadêmicos têm interesse em estudar e conhecer estas práticas durante a sua formação acadêmica, resultado este importante para a universidade planejar a inserção destas práticas que visam promover a saúde através da prevenção e o tratamento da saúde de forma integrativa e complementar, corroborando com as políticas de saúde previstas no país.

\section{REFERÊNCIAS}

1. Lima KMSV, Silva KL, Tesser CD. Práticas integrativas e complementares e relação com promoção da saúde: experiência de um serviço municipal de saúde. Interface: Comunicação Saúde Educação 2013;18:1-12. doi: http://dx.doi.org/10.1590/180757622013.0133

2. Nascimento MC, Romano VF, Chazan ACS, Quaresma CH. Formação em práticas integrativas e complementares em saúde: desafios para as universidades públicas. Rev Trab Educ Saúde 2018; 1-22. doi: http://dx.doi.org/10.1590/1981-7746-sol00130 3. Brasil.Portaria $n^{\circ} 702$, de 21 de março de 2018. Altera a Portaria de Consolidação $n^{\circ}$ 2/GM/MS, de 28 de setembro de 2017, para incluir novas práticas na Política Nacional de Práticas Integrativas e Complementares - PNPIC. Brasília, mar. 2018.

4. Teixeira MZ, Lin CA. Educação médica em terapêuticas não convencionais. Rev Med2013;92(4):224-35.doi: https://doi. org/10.11606/issn.1679-9836.v92i4p224-235

5. Santos LF, CunhaAZS. Autilização de práticas complementares por enfermeiros do Rio Grande do Sul. Rev Enferm - UFSM 2011;1(3):369-76. doi: http://dx.doi.org/10.5902/217976923047 6. Brasil. Resolução $n^{\circ} 971$, de 03 de maio de 2006. Aprova a Política Nacional de Práticas Integrativas e Complementares (PNPIC) no Sistema Único de Saúde. Brasília, mai. 2006.

7. Brasil. Portaria $n^{\circ} 849$, de 27 de março de 2017. Inclui a Arteterapia, Ayurveda, Biodança, Dança Circular, Meditação, Musicoterapia, Naturopatia, Osteopatia, Quiropraxia, Reflexoterapia, Reiki, Shantala, Terapia Comunitária Integrativa e Yoga à Política Nacional de Práticas Integrativas e Complementares. Brasília, mar. 2017.

8. Sirois FM., Salamonsen A, Kristoffersen AE. Reasons for continuing use of Complementary and Alternative Medicine (CAM) in students: a consumer commitment model. BMC Complementary and Alternative Medicine 2016;16(75):1-9. doi: https://doi.org/10.1186/s12906-016-1059-3

9. Caires JS, Andrade TA, Amaral JB, Calasans MTA, Rocha MDS. A utilização das terapias complementares nos cuidados paliativos: benefícios e finalidades. Cogitare Enferm 2014;19(3):514-20.doi: http//dx.doi.org/10.5380/ ce.v19i3.33861

10. Upchurch, DM, Rainisch BW. The importance of wellness among users of complementary and alternative medicine: findings from the 2007 National Health Interview Survey. BMC Complementary and Alternative Medicine 2015;15(362):1-10. doi: http//dx.doi.org/10.1186/s12906-015-0886-y

11. Bahall M.; Legall G. Knowledge, attitudes, and practices among health care providers regarding complementary and alternative medicine in Trinidad and Tobago. BMC Complementary and Alternative Medicine 2017;17(144):1-9. doi http//dx.doi.org/10.1186/s12906-017-1654-y

12. Machado MMT, Oliveira JC, Fechine ADL. Acupuntura: Conhecimento e Percepção de Professores Universitários. Rev Bras Educ Médica 2012;36(1):41-49. doi: http://dx.doi. org/10.1590/S0100-55022012000100006

13. Silva NCM, Iunes DH, Resck ZMR, Soares MI, Souza Junior DI, Vieira NF. Estratégias de ensino das terapias alternativas e complementares na graduação em Enfermagem. Rev Eletr Enf [Internet] 2013;15(4):1061-7. doi: http://dx.doi.org/10.5216/ree. v15i4.20568

14. Gomes DRGM, Almeida AMB, PessôaCKL, Porto CMV, França LC. A inclusão das terapias integrativas e complementares na formação dos acadêmicos da saúde. SANARE, Sobral 2017;16(Suppl 1):74-81.

15. Ribeiro LCC, Ribeiro M. Soares VAR. Avaliação acadêmica acerca das Diretrizes Curriculares Nacionais em saúde. Revistas Tempus - Actas de Saúde Coletiva 2015;9(1):167-87.doi: http:// dx.doi.org/10.18569/tempus.v9i1.1700

16. Brasil. Resolução n. 6, de 19 de outubro de 2017. Diretrizes Curriculares Nacionais do Curso de Graduação. Brasília, out. 2017.

17. Damasceno CM, Almeida JRGS, Dias CM, Dantas MGB, Saraiva SRGL, Andrade RB, Faria MD. Avaliação do conhecimento de estudantes universitários sobre medicina alternativa. Rev Baiana de Saúde Pública 2016;40(2):289-97. doi: http//dx.doi.org/10.22278/2318-2660

18. Salles LF, Homo RFB, Silva MJP. Práticas Integrativas e Complementares: Situação do seu Ensino na Graduação de Enfermagem no Brasil. Revista Saúde, UNG - SER. 2014;8(3/4):37-44.

19. Israel VL, Guimarães ATB, Pardo MB. Práticas Integrativas e Complementares em Saúde: Hidrotermalismo como Ambiente e Recurso de Atuação do Fisioterapeuta. Divers@ Revista Eletrônica Interdisciplinar [Internet] 2017;10(2):79-90. doi: http://dx.doi.org/10.5380/diver.v10i2.58063

20. Brasil. Resolução CFM n. ${ }^{\circ} 1000 / 80$ de 21 de julho de 1980. Acrescenta na relação de especialidades reconhecidas pelo CFM, para efeito de registro de qualificação de especialistas a hansenologia e a homeopatia. Brasília, jul. 1980.

21. Oliveira IF, Peluso BHB, Freitas FAC, Nascimento MC. Homeopatia na Graduação Médica: Trajetória da Universidade Federal Fluminense. Revista Brasileira de Educação Médica 2018;42(1):92-102. doi: http://dx.doi.org/10.1590/198152712015v42n1RB20160097

22. Hadley J, Hassan I, Khan KS. Knowledge and beliefs concerning evidence-based practice amongst complementary and alternative medicine health care practitioners and allied health care professionals: A questionnaire survey. BMC Complementary and Alternative Medicine 2008;8(45):1-7. doi: https://doi.org/10.1186/1472-6882-8-45

23. Tiralongo E, Wallis M. Attitudes and perceptions of Australian pharmacy students towards Complementary and Alternative Medicine - a pilot study. BMC Complementary and Alternative Medicine 2008;8(2):1-9. doi: https://doi.org/10.1186/1472$6882-8-2$

24. James PB, Bah A. Awareness, use, attitude and perceived need for Complementary and Alternative Medicine (CAM) education 
a descriptive cross-sectional survey. BMC Complementary and Alternative Medicine 2014;14(438):1-9.doi: https://doi. org/10.1186/1472-6882-14-438

23. Sousa IMC, Bodstein RCA, Tesser CD, Santos FAS, Hortale VA. Práticas integrativas e complementares: oferta e produção de atendimentos no SUS e em municípios selecionados. Caderno de Saúde Pública [online] 2012;28(11):2143-54.doi: http:// dx.doi.org/10.1590/S0102-311X2012001100014

24. Nascimento MVN, Oliveira IF. As práticas integrativas e complementares grupais e sua inserção nos serviços de saúde da atenção básica. Estudos de Psicologia 2016;21(3):272-281. doi: http://dx.doi.org/10.5935/1678-4669.20160026

Como citar: GOECKS, Débora Regina; MORSCH, Lisoni Muller; SILVA, Chana de Medeiros da. Formação de estudantes da área da saúde em práticas integrativas e complementares. Revista Interdisciplinar de Promoção da Saúde, Santa Cruz do Sul, v. 2, n. 2, june 2020. ISSN 2595-3664. Disponível em: <https://online.unisc.br/seer/index.php/ripsunisc/article/view/14080>. Acesso em: 02 jun. 2019. doi:https://doi.org/10.17058/rips.v2i2.14080 